\title{
Prevalence of Microalbuminuria in Hypertensive
}

\section{Patients}

\author{
Dr. Nilesh Gangadhar More ${ }^{* 1}$, Dr. Priyank Dumade ${ }^{2}$, Dr. Madhukar Gaikwad ${ }^{3}$, Dr. Navneet Agrahari ${ }^{4}$ \\ ${ }^{1,2}$ Assistant Professor, ${ }^{3}$ Associate Professor, ${ }^{4}$ Junior Resident \\ Department of Medicine Grant Government Medical College and Sir JJ Group of Hospitals, Byculla, Mumbai
}

\section{Corresponding Author -}

\section{Dr. Nilesh Gangadhar More}

Assistant Professor, Department of Medicine

Grant Government Medical College and Sir JJ Group of Hospitals, Byculla, Mumbai

\begin{abstract}
Summary
A total of 133 EssentialHypertension patients were studied.

Out of which 74(55.64\%) were male and 59(44.36\%) were female. Retinopathy was observed in 46(34.59\%) cases and Left Ventricular Hypertrophy by voltage criteria on ECG was seen in 36(27.07\%) patients. Out of 74 male patients $39(52.70 \%)$ were smokers while35 (47.30\%) were non-smokers. Microalbuminuria was seen in 54(40.60\%).Hypertension is an established risk factor for cardiovascular as well as renal disease. Prevalence of microalbuminuria in hypertensive patients suggests that screening for microalbuminuria is essential for intervention and prevention of further complications like end stage renal disease and cardiovascular disease and stroke. It is much more essential to screen for early nephropathy by testing microalbuminuria in hypertensive patients to prevent or halt the earliest stages of damage by vigorous control of hypertension. Further studies in large scale population are required for further verification of microalbuminuria as a screening tool to prevent progression of cardiovascular and renal disease.
\end{abstract}

\section{Introduction}

Hypertension is a major public health issue worldwide. The prevalence of hypertension in India is $25 \%$ in urban population and $10-15 \%$ in rural population, compared to west where it is $30 \% .^{[1]}$ Essential hypertension produces clinical proteinuria and a significant reduction in renal function in about $5-15 \%$ of patients. Several studies have shown that proteinuria and microalbuminuria are independent predictors of cardiovascular morbidity and mortality in patients with hypertension. ${ }^{[2,3]}$ Microalbuminuria is defined as urinary albumin excretion of $30-300 \mathrm{mg} / \mathrm{day}$, or $20-200 \mu \mathrm{g} / \mathrm{min}$ and it is an earlier sign of vascular damage, also it is a marker of general vascular dysfunction and is considered a predictor of worse outcomes for both kidney and heart patients. Possible mechanisms for albuminuria in hypertensive patients are augmented glomerular hydrostatic pressure and altered permeability of glomerular basement membrane. Urinary albumin excretion (UAE) in hypertensive patients is a continuous and progressive marker of renal damage and cardiovascular events. ${ }^{[4]}$ Also target organ damage is more frequent in patients with microalbuminuria. ${ }^{[5]}$ Various factors can affect the prevalence of microalbuminuria in hypertension which includes age, sex, race, severity of the disease, and concomitant risk factors, which accounts for the large differences in the prevalence of microalbuminuria, with prevalence rates going from a low of $4.7 \%$ to a high of $46 \%$. About $25 \%$ of patients with end-stage renal disease have hypertension as the primary diagnosis. ${ }^{[6]}$ The main determinant of albumin excretion rate in patients with mild hypertension without cardiovascular complications seems to be the hemodynamic load, whereas in subjects with more severe hypertension and associated target organ damage, the augmented urinary albumin leak is probably the consequence of glomerular damage. ${ }^{[7]}$ Two mechanisms have been proposed for the greater urinary albumin excretion (UAE) in some patients with essential hypertension: (i) increased glomerular hydrostatic pressure, (ii) increased selectivity of the glomerular basement membrane. In hemodynamic mechanism glomerular hydrostatic pressure is regulated by the relative vasodilatation of the afferent and vasoconstriction of the efferent glomerular arterioles. The tone of these arterioles is regulated by different mechanisms, the efferent arteriole is more sensitive to the pressor action of angiotensin II. $^{[8]}$ Whereas the afferent arteriole is more sensitive to the vasodilator action of atrial natriuretic peptide. ACE 


\section{International Journal of Innovative Research in Medical Science (IJIRMS) \\ Volume 03 Issue 05 May 2018, ISSN No. - 2455-8737 \\ Available online at - www.ijirms.in}

inhibitors preferentially dilate the efferent arterioles. ${ }^{[9]}$ An elevation of systemic arterial pressure is associated with vasoconstriction of the glomerular afferent arterioles, which prevents transmission of the elevated hydrostatic pressure to the glomerulus and maintains the glomerular hydrostatic pressure. $^{[10]}$

In patients with essential hypertension, the combined presence of microalbuminuria and hyperlipidemia is frequent. A possible cause for the association between microalbuminuria and hyperlipidemia could be an abnormal intake of lipids with the diet. ${ }^{[11]}$ Microalbuminuria is independent risk factor for hyperlipidemia, atherosclerosis, ischemic heart disease and heart failure. Also, hypertensive patients with microalbuminuria have significantly higher risk of future cerebrovascular events as compared to normoalbuminuric patients. Microalbuminuria is proportional to severity and duration of essential hypertension. Thus, early screening for microalbuminuria in hypertensive patients with aggressive BP control may help decrease the ongoing target organ damage. This study looks into prevalence of this important parameter in essential hypertension patients.

\section{Design}

This is an observational, cross-sectional study in hypertensive patients.

\section{Material and Methods}

The study was carried out at Grant Government Medical College and Sir JJ group of Hospitals, Byculla, Mumbai in the Out Patient Clinic of General Medicine Dept. Patients in the age group of 18-70 yrswho recorded a high blood pressure based on JNC 8 (Eighth Report of the Joint National Committee on Prevention, Detection, Evaluation and Treatment of High Blood Pressure) criteria during three consecutive visits to the outpatient clinic, diagnosed essential hypertension patients who were on antihypertensive treatment (other than ACE inhibitors/Angiotensin receptor blockers) and who had a creatinine clearance greater than $80 \mathrm{ml} / \mathrm{min} / 1.73 \mathrm{~m} 2$, were included in the study. A total of 133essential hypertension patients were included in the study. The random sample selection method was simple; patients following in the outpatient clinic who were found eligible were serially included for evaluation of microalbuminuria.

\section{Exclusion criteria}

Patients who had been treated with angiotensin-converting enzyme inhibitors or angiotensin II receptor antagonists (ARBs) during at least thepreceding2 weeks were excluded. Patients with Diabetes Mellitus, secondary hypertension, BP $\geq 210 / 110 \mathrm{~mm} \mathrm{Hg}$, unstable hypertension, hyperkalemia
$>5.5 \mathrm{mEq} / \mathrm{L}$, hypokalemia $<3.0 \mathrm{mEq} / \mathrm{Lwere}$ excluded. Patients with clinical or laboratory evidence of hepatic, renal, thyroid, or any other major disease, overt proteinuria, women on birth control pills, and pregnant women were excluded. Renal cause for hypertension was excluded based on results of normal urinalysis, serum creatinine, serum electrolytes, creatinine clearance, and renal ultrasonography.

Blood pressure measurement was carried out according to the WHO guidelines. ${ }^{[12]}$ Blood pressure was measured after five minutes of rest using a mercury sphygmomanometer, the values reported were the average of three consecutive measurements taken over a 15-minute period. (Mean arterial pressure was calculated adding diastolic blood pressure with one-third of pulse pressure.) Demographic data, age, sex, weight, associated cardiovascular disease, and clinical parameters were all recorded. All routine biochemical tests were performed using laboratory autoanalyser and microalbuminuria tests were performed by the immune turbidimetry assay method.

\section{Statistical analysis}

All reports were entered in Excel sheet (Windows office 2013).A statistical analysis was performed by using standard methods to calculate rates and proportions; $\mathrm{Z}$ test was used for analyzing the differences between the variables. A total $\mathrm{P}$ value was used for calculating statistical significance. A $\mathrm{P}<0.05$ was considered as statistically significant.

\section{Observation and Results}

A total of 133 patients were included in the study. Out of 133 study patients $74(55.64 \%)$ were male and $59(44.36 \%)$ were female. Out of 74 male patients $39(52.70 \%)$ were smokers while $35(47.30 \%)$ were non-smokers.

Retinopathy was observed in $46(34.59 \%)$ cases. Left Ventricular Hypertrophy by ECG criteria was seen in $36(27.07 \%)$ patients.

Out of 133 hypertensive patients, 103(77.44\%) were classified as JNC 8 Stage 1 and the remaining 30(22.56\%) as Stage 2. Out of 103 Stage 1 hypertensive patients $31(30.10 \%)$ had microalbuminuria. Out of 30Stage 2 hypertensive patients, 23(76.66\%) had microalbuminuria. Thus, overall microalbuminuria was seen in 54(40.60\%) patients. Microalbuminuria was significantly higher in patients with Stage 2 hypertension $(\mathrm{P}<0.05)$. The systolic $\mathrm{BP}$, diastolic BP and BMI were significantly higher in the microalbuminuria group.

On further comparison, serum urea and serum creatinine were significantly higher in microalbuminuria group. Similarly, microalbuminuric patients had significantly high cholesterol. 
International Journal of Innovative Research in Medical Science (IJIRMS)

Volume 03 Issue 05 May 2018, ISSN No. - 2455-8737

Available online at - www.ijirms.in

Table1: Showing Sex percentage, retinopathy, LVH and smoking habits

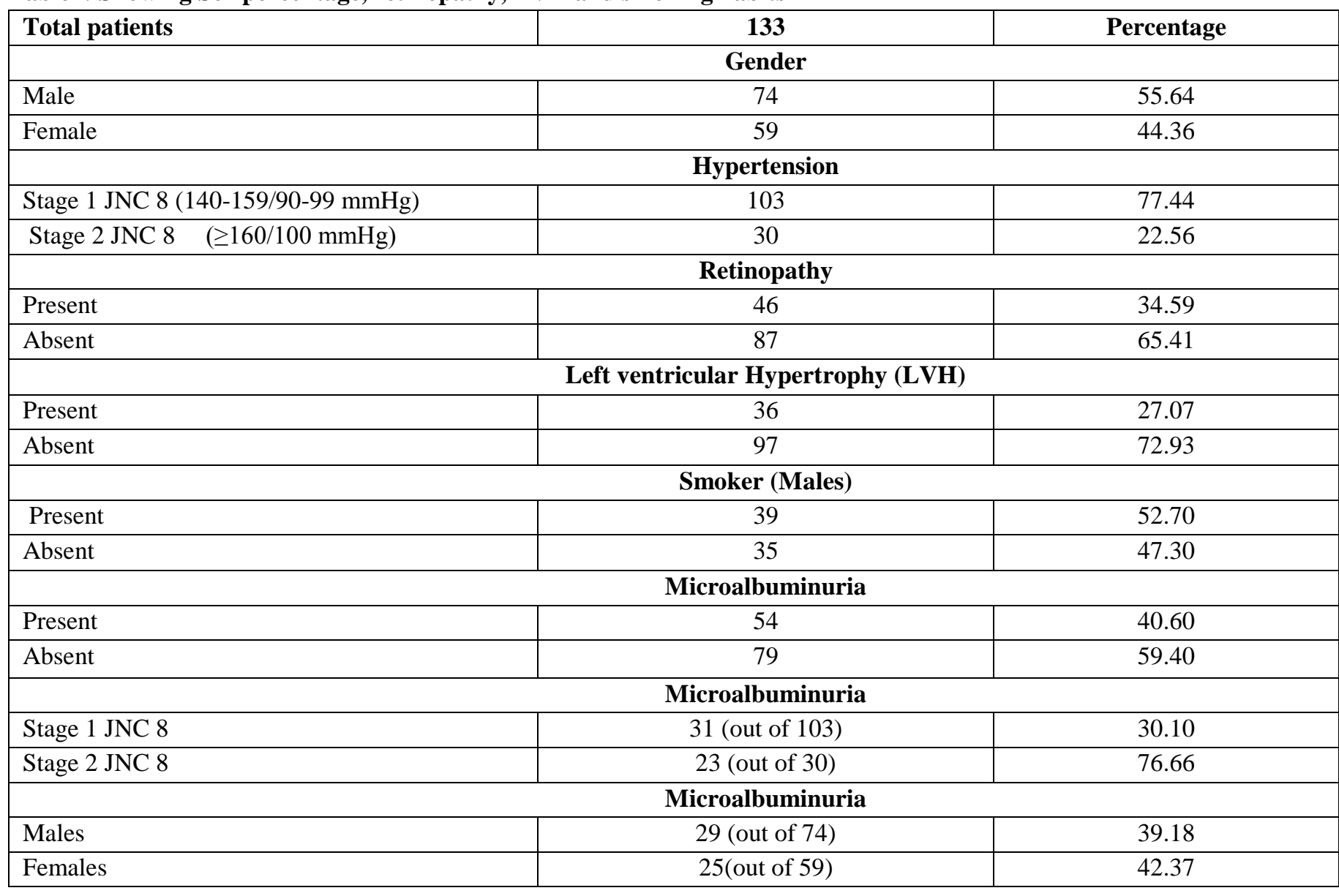

Table 2: Various parameters in microalbuminuric and normal albuminuric patients

\begin{tabular}{|l|c|c|c|c|c|}
\hline Parameters & \multicolumn{2}{|c|}{$\begin{array}{c}\text { Microalbuminuric cases } \\
(\mathbf{n = 1 3 3 )}\end{array}$} & $\begin{array}{c}\text { Normal albumin excretion } \\
(\mathbf{n = 2 2 6})\end{array}$ & P value \\
\hline \multirow{2}{*}{ Mean age } & Mean & SD & Mean & SD & \\
\hline Systolic Blood pressure (mmHg) & 45.57 & 8.4 & 39.73 & 9.6 & 0.035 \\
\hline Diastolic Blood pressure (mmHg) & 161.25 & 20.36 & 146.23 & 18.52 & $<0.00001$ \\
\hline BMI & 105.63 & 9.65 & 87.56 & 12.54 & $<0.00001$ \\
\hline Serum Urea (mg/dL) & 29.56 & 2.13 & 27.58 & 1.45 & $<0.00001$ \\
\hline Serum Creatinine (mg/dL) & 36.54 & 7.56 & 34.12 & 7.41 & $<0.00001$ \\
\hline Serum uric acid (mg/dL) & 0.91 & 0.19 & 0.86 & 0.16 & 0.000252 \\
\hline Serum Cholesterol (mg/dL) & 4.45 & 0.97 & 4.41 & 0.77 & 0.555995 \\
\hline Urinary albumin excretion (mg/ 24 hours) & 195.56 & 39.25 & 179.23 & 35.56 & $<0.00001$ \\
\hline
\end{tabular}

\section{Discussion}

The study was conducted in Out Patient Clinic in Dept. of Medicine at Grant Government Medical College and Sir JJ group of Hospitals, Byculla, Mumbai. There is evidence that, as in diabetes, already modestly increased levels of albumin excretion are associated with an increased GFR in patients with essential hypertension. Microalbuminuria is associated with an enhanced risk for cardiovascular morbidity and mortality, with an enhanced risk for progressive renal failure in hypertensive subjects. ${ }^{[13]}$ Therefore screening for microalbuminuria may be an excellent tool, either alone or in combination with screening for hypertension and hypercholesterolemia. ${ }^{[14]}$ Our study shows that microalbuminuria is associated with higher systolic as well as diastolic blood pressure. This echoes with other studies that have shown that there is an association between microalbuminuria and high levels of blood pressure. Ahmedani et al. showed microalbuminuria positive group had a higher systolic and diastolic blood pressure compared to microalbuminuria negative group $(\mathrm{p}<0.001){ }^{[15]}$ Arkedani et al. and Varghese et al. in their study showed a statistical correlation between the prevalence of microalbuminuria and the diastolic blood pressure. ${ }^{[16,17]}$ Svenson et al. reported that high blood pressure increased the risk of developing signs of nephropathy. ${ }^{[18]}$ Pasko et al found that microalbuminuric patients had higher systolic and diastolic blood pressure. ${ }^{[19]}$ Significant statistical correlation 


\section{International Journal of Innovative Research in Medical Science (IJIRMS) \\ Volume 03 Issue 05 May 2018, ISSN No. - 2455-8737 \\ Available online at - www.ijirms.in}

between hypertension and microalbuminuria was found in our study. Hypertension can cause microalbuminuria and hypertensive nephropathy that can accelerate the progression of diabetic nephropathy. A higher level of systolic blood pressure and diastolic blood pressure in suggests that hypertension is associated with microalbuminuria. ${ }^{[20]}$ Microalbuminuria should be tested routinely in hypertensive patients because its appearance, progression to proteinuria, or regression to normal albuminuria can be correlated with a higher or lower risk of coronary heart disease, stroke, or peripheral vascular disease. That means urinary albumin excretion is a marker of cardiovascular risk, and also a marker of treatment efficacy. The prevalence of microalbuminuria found in this sample of hypertensive patients,54/133(40.60\%), suggests that this cardiovascular risk factor is very common in clinical cardiology A similar finding was shown by Poudel $\mathrm{B}$ et al in their study percentage of microalbuminuria in essential hypertensive patients was $51.88 \% .{ }^{[21]}$ Some study had showed approximately $30 \%$ of hypertensive patients had microalbuminuria. ${ }^{[22]}$ Study by Bramlage $\mathrm{P}$ et al documented prevalence of $21.2 \%$ of microalbuminuria in patients with hypertension. ${ }^{[23]}$ While Hillege $\mathrm{HL}$ et al. found that microalbuminuria is common in a non-diabetic, non hypertensive population, and an independent indicator of cardiovascular risk factors and cardiovascular morbidity. ${ }^{[24]}$

It has been shown in several studies that microalbuminuria detection is a first-line tool for identifying hypertensive patients who are at a high cardiovascular risk and this had been proven in our study by inferring that UAE alone had shown a statistical significant difference between patients with microalbuminuria and those with normal albumin, whereas other serological parameters like uric acid did not show any statistical significant difference between these two groups but serum cholesterol, serum urea, serum creatinine and urinary albumin excretion showed statistical significant difference between these two groups. A study by Marudhaiveeran et al. also showed significant difference between urinary albumin excretion but he did not find significance in serum urea, creatinine, uric acid and cholesterol. $^{[25]}$ According to study up to $80 \%$ of hypertensive patients show a concomitant cardiovascular risk factor. In our study higher levels of creatinine with the increased level of urinary microalbumin may be due to subclinical changes in the glomeruli of hypertensive patients. ${ }^{[26]}$ Proteinuria represents a predictor of progressive renal impairment in all form of glomurulopathies ${ }^{[27]}$ Some studies stated that the presence of microalbuminuria in early stage of essential hypertension can be taken as an important predictor for progression of renal disease. ${ }^{[28]}$

\section{Conclusion}

Hypertension is an established risk factor for cardiovascular as well as renal disease. Prevalence of microalbuminuria in hypertensive patients suggests that screening for microalbuminuria is essential for intervention and prevention of further complications like end stage renal disease and cardiovascular disease and stroke. It is much more essential to screen for early nephropathy by testing microalbuminuria hypertensive patients to prevent or to retard the earliest stages of damage by vigorous control of hypertension. Further studies in large scale populations are required for verification of microalbuminuria as a screening tool to prevent progression of cardiovascular and renal disease.

\section{References}

[1] Epidemiology of hypertension. JAPI 2013; 61:123.

[2] Verdecchia P, Reboldi GP. Hypertension and microalbuminuria: The new detrimental duo. Blood Press 2004; 13:198-211.

[3] Bianchi S, Bigazzi R, Baldari G, Campese VM. Microalbuminuria in patients with essential hypertension: Effects of several antihypertensive drugs. Am J Med 1992; 93:525-8.

[4] Gerstein, H.C., Mann, J.F.E., Yi, Q. et al. Albuminuria and risk of cardiovascular events, death, and heart failure in diabetic and non diabetic individuals. JAMA. 2001;286: 421-426

[5] Jensen, J.S., Feldt-Rasmussen, B., Borch-Johnsen, K. et al. Microalbuminuria and its relation to cardiovascular disease and risk factors. A population-based study of 1254 hypertensive individuals. J Hum Hypertens. 1997; 11: 727-732

[6] Bigazzi R, Bianchi S, Campese VM, Baldari G. Prevalence of microalbuminuria in a large population of patients with mild to moderate essential hypertension. Nephron 1992; 61:94-7.

[7] Palatini P1.Microalbuminuria in hypertension. Curr Hypertens Rep. 2003 Jun;5(3):208-14

[8] Edwards RM: Segmental effects of norepinephrine and angiotensin II on isolated renal microvessels. Am J Physiol 1983;244:F526-F534

[9] Raij L, Chiou X, Owens R, Wrigley B: Therapeutic implication of hypertensioninduced glomerular injury: Comparison of enalapril, combinationofhydralazinereserpine hydrochlorothiazide. Am J Med 1985;9:37- 41

[10] Hostetter TH, Olson JL, Rennke HG, Venkatachalam MA, Brenner BM: Hyperfiltration in remnant nephrons: A potential adverse response to renal ablation. Am J Physiol 1981241:F85-F93

[11] Kannel WB, Stampfer M William P. Castelli M, Joel V. The prognostic significance of proteinuria: The Framingham Study. Am Heart , 1984;108:1347-1352

[12] 1999 World Health Organization International Society of Hypertension Guidelines for the 
management of Hypertension. J Hypertens. 1999; 17: $151-183$

[13] Paul E. de Jong, Hans L. Hillege, Sara Joan PintoSietsma, Dick de Zeeuw; Screening for microalbuminuria in the general population: a tool to detect subjects at risk for progressive renal failure in an early phase?, Nephrology Dialysis Transplantation, Volume 18, Issue 1, 1 January 2003, Pages 10-13,

[14] Scheid DC, McCarthy LH, Lawler FH et al. screening for microalbuminuria to prevent nephropathy in patients with diabetes. J Fam Pract2001; 50:661-668

[15] Ahmadani MY, Fawwad A, Basit A, Hydrie ZI. Microalbuminuria prevalence study in hypertensive patients with type 2 diabetes in Pakistan. J Ayub Med Coll Abbottabad. 2008 Jul-Sep; 20(3):117-20.

[16] Afkhami-Ardekani M, Modarresi M, Amirchaghmaghi E. Prevalence of microalbuminuria and its risk factors in type 2 diabetic patients. Indian J Nephrol. 2008 Jul; 18(3):112-7.

[17] Varghese A, Deepa R, Rema M, Mohan V Prevalence of microalbuminuria in type 2 diabetes mellitus at a diabetes centre in southern India. Postgrad Med J. 2001 Jun; 77(908):399-402.

[18] Svensson M, Sundkvist G, Arnqvist HJ, Björk E, Blohmé G, Bolinder J, Henricsson M, Nyström L, Torffvit O, Waernbaum I, Ostman J, Eriksson JW, Diabetes Incidence Study in Sweden (DISS). Signs of nephropathy may occur early in young adults with diabetes despite modern diabetes management: results from the nationwide population-based Diabetes Incidence Study in Sweden (DISS).Diabetes Care. 2003 Oct; 26(10):2903-9.

[19] Pasko N, Toti F, Zekollari E, Strakosha A, Kacori $\mathrm{V}$, Thereska N. Prevalence of microalbuminuria in type 2 diabetic patients in Tirana, a preliminary multicenter study. J DiabMell. 2013; 3:145-149. DOI: $10.4236 / j \mathrm{dm} .2013 .33022$.

[20] Sigdel M, Rajbhandari N, Basnet S, Nagila A, Basnet P, Tamrakar BK Microalbuminuria among type-2 diabetes mellitus patients in Pokhara, Nepal. Nepal Med Coll J. 2008 Dec; 10(4):242-5.

[21] Bibek Poudel, Binod Kumar Yadav, Ashwini Kumar Nepal, Bharat Jha, Kanak Bahadur Raut. Prevalence and Association of Microalbuminuria in Essential Hypertensive Patients. N Am J Med Sci. 2012 Aug; 4(8): 331-335. doi: 10.4103/19472714.99501

[22] Schrader J, Lüders S, Kulschewski A, Hammersen F, Züchner C, Venneklaas U, Schrandt G, Schnieders M, Rangoonwala B, Berger J, Dominiak P, Zidek W, MARPLE Study Group. Microalbuminuria and tubular proteinuria as risk predictors of cardiovascular morbidity and mortality in essential hypertension: final results of a prospective long-term study (MARPLE Study)*.J Hypertens. 2006 Mar; 24(3):541-8.

[23] Bramlage P, Pittrow D, Lehnert H, Höfler M, Kirch W, Ritz E, Wittchen HU. Frequency of albuminuria in primary care: a cross-sectional study.Eur J Cardiovasc Prev Rehabil. 2007 Feb; 14(1):107-13.

[24] Hillege HL, Janssen WM, Bak AA, Diercks GF, Grobbee DE, Crijns HJ, Van Gilst WH, De Zeeuw D, De Jong PE, Prevend Study Group. Microalbuminuria is common, also in a nondiabetic, nonhypertensive population, and an independent indicator of cardiovascular risk factors and cardiovascular morbidity.J Intern Med. 2001 Jun; 249(6):519-26.

[25] Marudhaiveeran GM, Radhakrishnan S, Alphonse F. Prevalence of microalbuminuria among patients with essential hypertension. Trop J Med Res 2014; $17: 76-80$

[26] Rodilla E, Pérez-Lahiguera F, Costa JA, González C, Miralles A, Moral D, Pascual JM Association between serum uric acid, metabolic syndrome and microalbuminuria in previously untreated essential hypertensive patients. MedClin (Barc). 2009 Jan 17; 132(1):1-6.

[27] Maschio G, Oldrizzi L, Rugiu C, Valvo E, Lupo A, Loschiavo C, Tessitore N, Fabris A, Gammaro L, Panzetta G. Factors affecting progression of renal failure in patients on long-term dietary protein restriction. Kidney Int Suppl. 1987 Oct; 22():S4952.

[28] Pruijm MT, Madeleine G, Riesen WF, Burnier M, Bovet P. Prevalence of microalbuminuria in the general population of Seychelles and strong association with diabetes and hypertension independent of renal markers. J Hypertens. 2008 May; 26(5):871-7. 